\title{
Predictive Value of Cerebrovascular Reserve in Patients with Carotid Artery Stenosis for Choosing Treatment Strategy
}

\author{
Aliev VA ${ }^{1 *}$, Semenyutin VB' and Panuntsev $G K^{2}$ \\ ${ }^{1} 2^{\text {nd }}$ Department of Neurosurgery, Municipal Hospital of Saint Martyr Elizabeth, Saint-Petersburg, Russia \\ ${ }^{2}$ Laboratory of Brain Circulation Pathology, Almazov National Medical Research Center, Saint-Petersburg, \\ Russia
}

*Corresponding author: Aliev Vugar Ali Ogli, PhD, MD, Head of the 2nd Department of Neurosurgery, Municipal Hospital of Saint Martyr Elizabeth, 190000, Saint-Petersburg, 14 Vavilovih str, Russia, Tel: +79531577423

\begin{abstract}
The choice of treatment of carotid artery stenosis is firstly based on assessment of the degree of stenosis and embologeneity of the atherosclerotic plaque. But even in "good-risk" population it is still difficult to identify the patients who are particularly at high threat of stroke and would most benefit from carotid reconstruction. Along with common criteria numerous studies also focus on a prognostic role of a cerebrovascular reserve (CVR) and its state which allows to carry out more objective and pathogenetically substantiated selection of cases for surgical and medical treatment. The present study is a literature review of CVR assessment with different methods in relation to the clinical course of disease, severity of stenosis, the state of collateral circulation, its dynamics after operative procedures. Questions are discussed about the informative value of CVR indices in predicting ischemic strokes and postoperative hyperperfusion complications. It was concluded there is a great need for carrying out multicenter randomized studies to determine the prognostic role of CVR in the choice of treatment strategy, to clarify the indications and evaluate the effectiveness of surgical intervention in patients with stenotic lesions of the internal carotid arteries.
\end{abstract}

\section{Keywords}

Carotid artery stenosis, Cerebral autoregulation, CO2reactivity, Stenting/endarterectomy, Transcranial doppler, Treatment strategy

\section{List of Abbreviations}

ASP: Atherosclerotic Plaque; BFV: Blood Flow Velocity; CT: Computed Tomography; CVR: Cerebrovascular Reserve; ICA: Internal Carotid Artery; MCA: Middle Cerebral Artery; MR-Angiography: Magnetic Resonance Angiography; MRI:
Magnetic Resonance Imaging; PET: Positron Emission Tomography; SPECT: Single-Photon Emission Computed Tomography; TCD: Transcranial Doppler Sonography

\section{Introduction}

Numerous randomized clinical studies have proved high efficacy of surgical treatment of symptomatic internal carotid artery (ICA) stenosis as means of prevention of recurrent strokes in the ipsilateral region of intracranial cerebral arteries. As for cases with an asymptomatic clinical course of ICA stenosis, an advantage of surgical intervention over optimized medical therapy is still in question $[1,2]$. The main criteria in choosing treatment strategy are a degree of ICA stenosis and morphologic characteristics of an atherosclerotic plaque (first of all its embologeneity) which causes narrowing of an artery and thromboembolism of major intracranial arteries $[3,4]$. Other equally important determinants include various comorbid risk factors (myocardial infarction, cardiac rhythm disorders, diabetes mellitus, obesity, etc.) leading to an inevitable increase of a rate of periprocedural cerebral and cardiologic complications and unfavorable outcomes after surgery [5]. However, one will face a certain problem even in cases with "a minimum set" of risk factors as it is rather difficult to identify who of them have a really high threat of stroke and, as a result, a greater need for reconstructive operation on ICA. Along with common criteria based on data of conventional neurovisualization (spiral CT, MRI

Citation: Aliev VA, Semenyutin VB, Panuntsev GK (2019) Predictive Value of Cerebrovascular Reserve in Patients with Carotid Artery Stenosis for Choosing Treatment Strategy. Int J Pathol Clin Res 5:086. doi.org/10.23937/2469-5807/1510086

Accepted: April 06, 2019: Published: April 08, 2019

Copyright: (C) 2019 Aliev VA, et al. This is an open-access article distributed under the terms of the Creative Commons Attribution License, which permits unrestricted use, distribution, and reproduction in any medium, provided the original author and source are credited. 


\begin{tabular}{|c|c|c|c|c|c|c|c|c|c|c|}
\hline 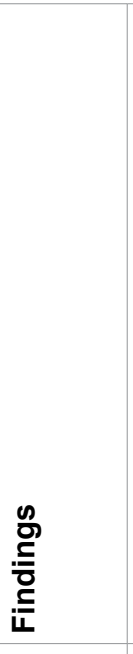 & 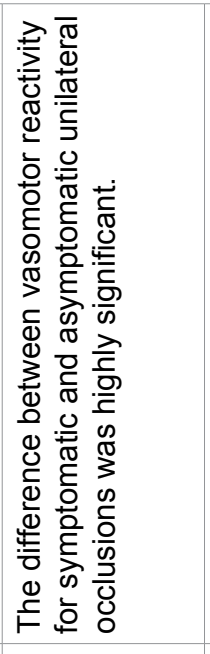 & 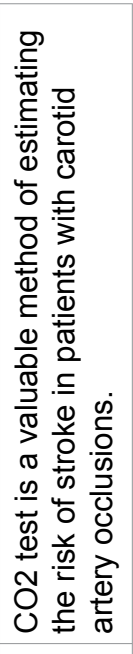 & 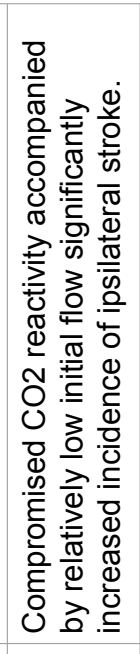 & 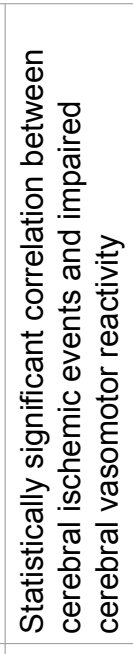 & 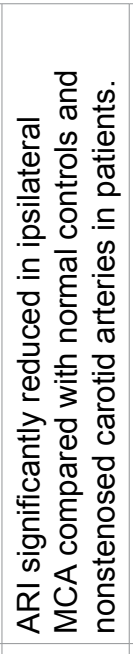 & 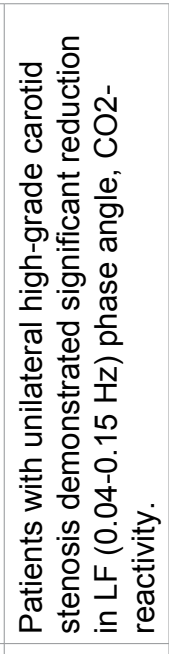 & 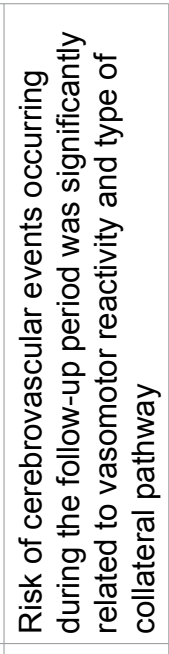 & 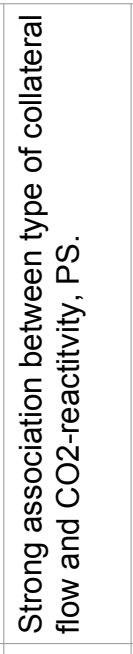 & 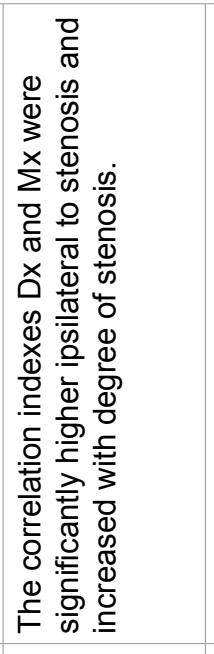 & 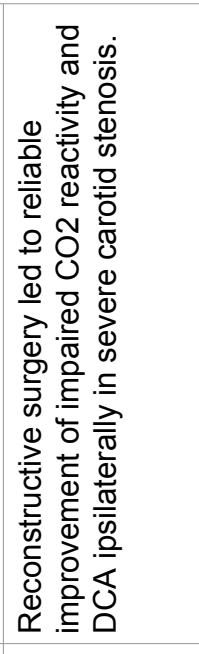 \\
\hline 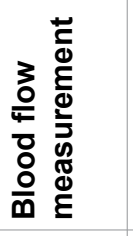 & Oि & Oि & $\frac{\mathfrak{\varrho}}{\mathscr{Q}}$ & Oि & Oि & $\underset{P}{\stackrel{O}{O}}$ & Oि & $\begin{array}{l}O \\
\stackrel{\rho}{1}\end{array}$ & $\stackrel{\bigcirc}{O}$ & Oि \\
\hline 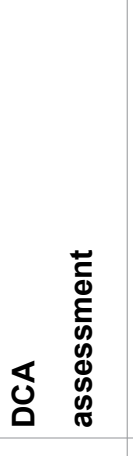 & 1 & 1 & 1 & 1 & 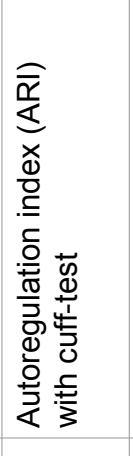 & 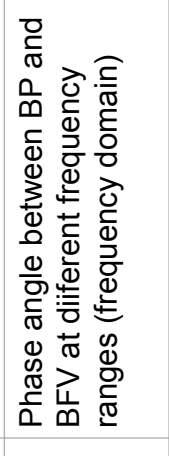 & 1 & 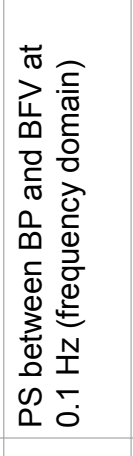 & 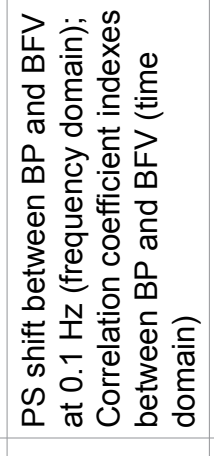 & 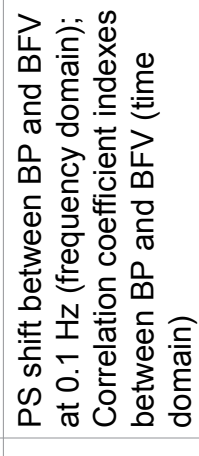 \\
\hline 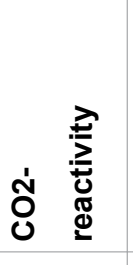 & 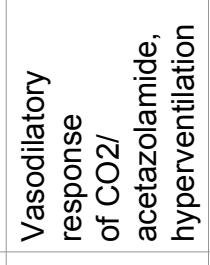 & 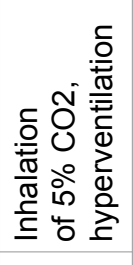 & 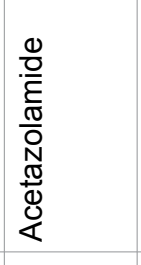 & 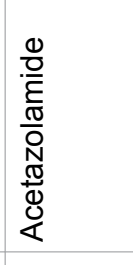 & 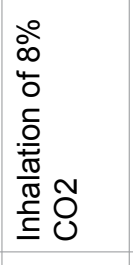 & 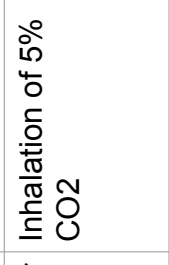 & 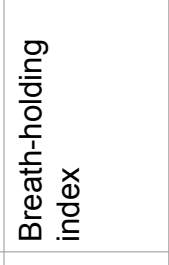 & 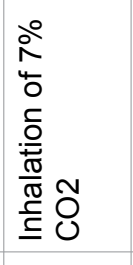 & 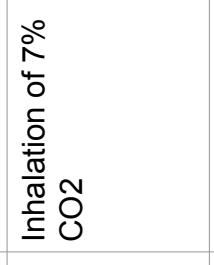 & 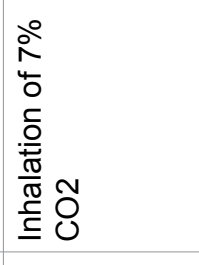 \\
\hline 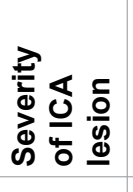 & $\begin{array}{l}\frac{.}{0} \\
\frac{0}{09} \\
\frac{D}{0} \\
0 \\
0\end{array}$ & & 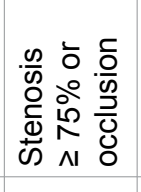 & 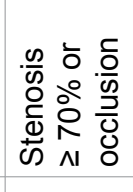 & 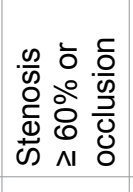 & 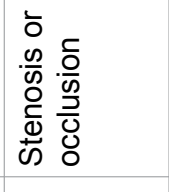 & & 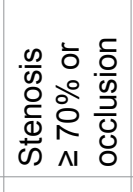 & 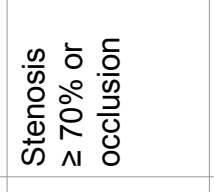 & $\begin{array}{l}1 \\
\frac{0}{\omega} \\
0 \\
\stackrel{5}{\Phi} \\
\dot{\Phi} \\
\dot{\omega}\end{array}$ \\
\hline 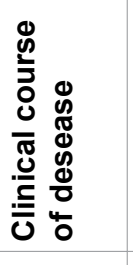 & 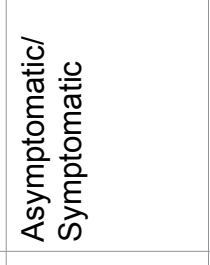 & 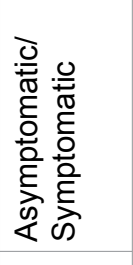 & 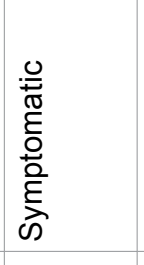 & 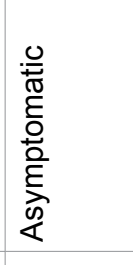 & 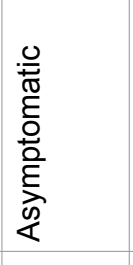 & 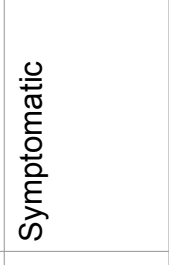 & 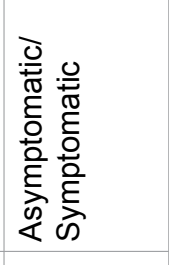 & 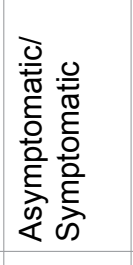 & 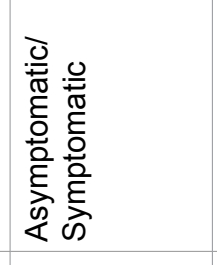 & 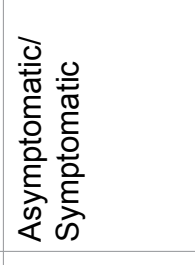 \\
\hline 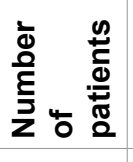 & 움 & $\infty$ & $\begin{array}{l}\infty \\
\bullet\end{array}$ & $\underset{\forall}{\forall}$ & $\hat{\sim}$ & $\mathscr{\infty}_{\infty}^{\infty}$ & 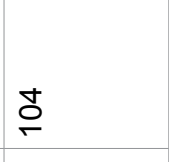 & $\stackrel{5}{\circ}$ & $\stackrel{\mathscr{m}}{\sim}$ & 冓 \\
\hline 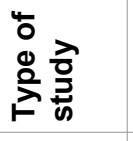 & $\begin{array}{l}\frac{t}{0} \\
\frac{0}{0} \\
0\end{array}$ & $\begin{array}{l}\frac{\pi}{0} \\
\frac{0}{0} \\
0\end{array}$ & $\begin{array}{l}\frac{\pi}{0} \\
\frac{0}{0} \\
0\end{array}$ & $\frac{\pi}{0}$ & $\begin{array}{l}\frac{\pi}{0} \\
\frac{1}{0} \\
0\end{array}$ & $\begin{array}{l}\frac{\pi}{0} \\
\frac{0}{0} \\
0\end{array}$ & $\begin{array}{l}\frac{t}{0} \\
\frac{0}{0} \\
0\end{array}$ & $\frac{\pi}{0}$ & $\begin{array}{l}\frac{\pi}{0} \\
\frac{7}{0} \\
0\end{array}$ & $\begin{array}{l}\frac{\pi}{0} \\
\frac{0}{0} \\
0\end{array}$ \\
\hline 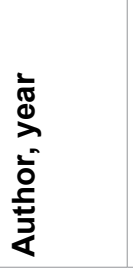 & 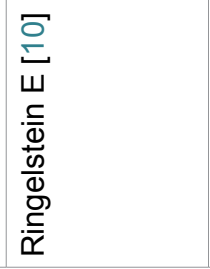 & 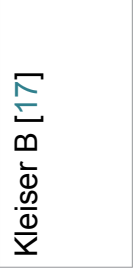 & 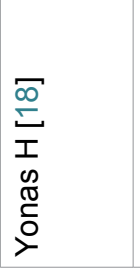 & $\begin{array}{l}\frac{\sigma}{5} \\
\frac{1}{4} \\
\vdots \\
0\end{array}$ & 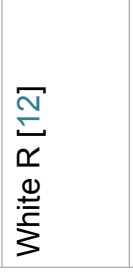 & 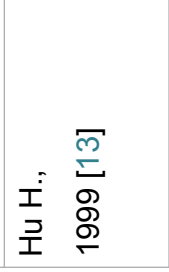 & 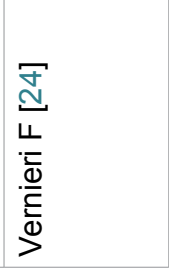 & 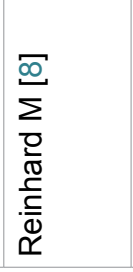 & 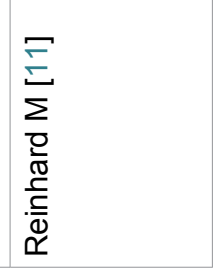 & 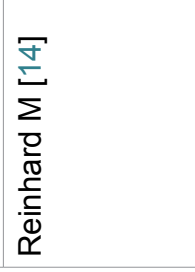 \\
\hline
\end{tabular}




\begin{tabular}{|c|c|c|c|c|}
\hline 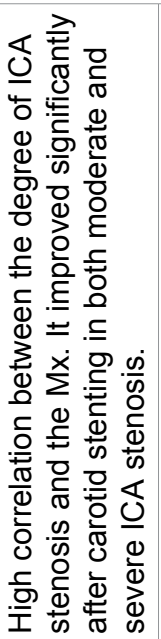 & 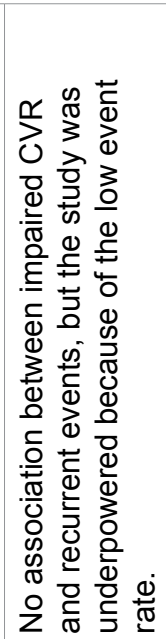 & 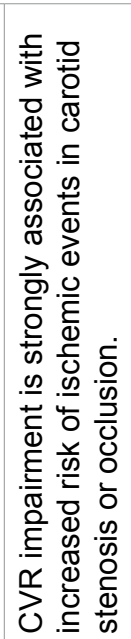 & 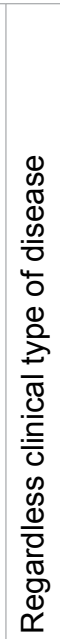 & 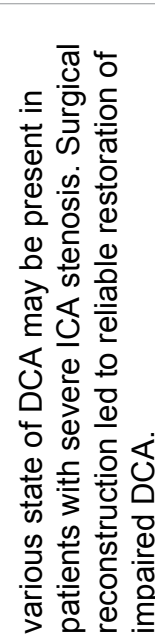 \\
\hline
\end{tabular}

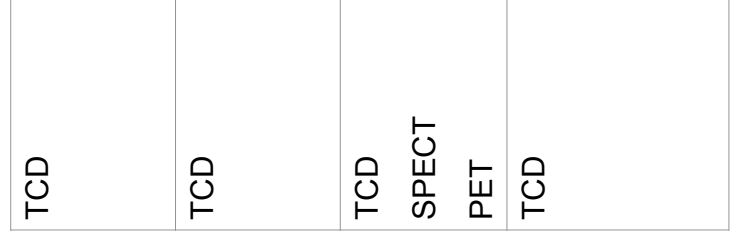

\begin{tabular}{|c|c|c|c|}
\hline 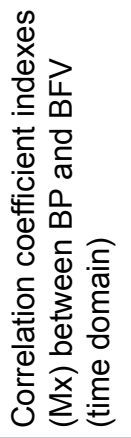 & 1 & 울 & 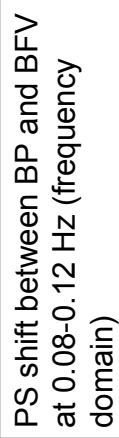 \\
\hline 1 & 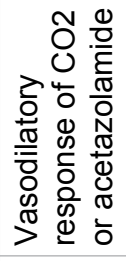 & 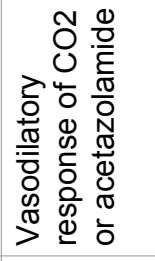 & 1 \\
\hline 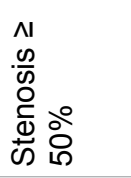 & 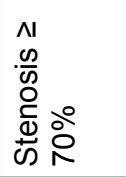 & 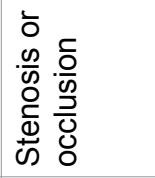 & 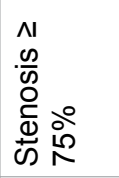 \\
\hline
\end{tabular}

\begin{tabular}{|c|c|c|c|}
\hline 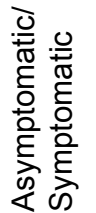 & 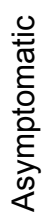 & 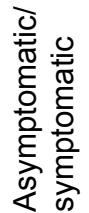 & 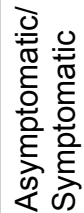 \\
\hline
\end{tabular}

\begin{tabular}{|c|c|c|c|}
\hline$\check{\sim}$ & 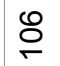 & চু & $\stackrel{10}{m}$ \\
\hline
\end{tabular}

\begin{tabular}{|c|c|c|c|c|}
\hline $\begin{array}{l}\frac{T}{0} \\
\text { 응 } \\
\text { Oे }\end{array}$ & 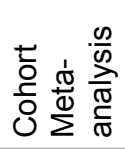 & 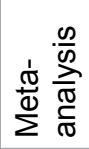 & $\begin{array}{l}\frac{T}{0} \\
\text { 잉 } \\
\text { U }\end{array}$ & 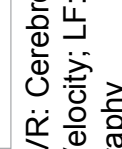 \\
\hline 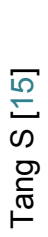 & 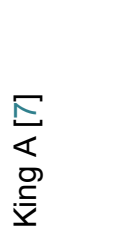 & $\begin{array}{l}\sigma \\
\frac{0}{\pi} \\
\frac{\pi}{2} \\
\frac{0}{7} \\
0\end{array}$ & 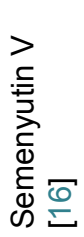 & 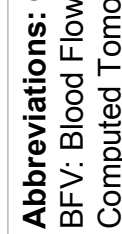 \\
\hline
\end{tabular}

and MR-angiography, catheterization X-ray cerebral angiography) some studies also focus on a prognostic role of a cerebrovascular reserve (CVR) and its state which allows to carry out more objective and pathogenetically substantiated selection of cases for surgical and medical treatment $[6,7]$.

The present study is a literature review of CVR assessment with different methods in relation to the clinical course of disease, severity of stenosis, the state of collateral circulation, its dynamics after operative procedures. We analyzed the most important publications on the problem of CVR in patients with stenosis and occlusion of the carotid arteries that were published from January 1988 up to December 2017 and available in Pubmed/Medline database (Table 1). We used the search terms 'cerebrovascular reserve', 'dynamic autoregulation', 'transfer function analysis', 'correlation analysis', 'carotid stenosis', 'occlusion', 'endovascular', 'endoarterectomy', 'stroke' and their synonyms.

\section{Pathophysiologic Aspects of Cerebral Hemo- dynamics Impairment and Methods of Study- ing of Cerebrovascular Reserve}

CVR reduction is one of the key pathophysiologic mechanisms responsible for a higher rate of ischemic events in cases with stenosic and occlusive lesions of ICA $[6,8,9]$. Marked ICA stenosis results in considerable decrease of cerebral perfusion pressure in the ipsilateral region up to a lower threshold of autoregulation. Thus, compensatory vasodilation under these conditions as well as in response to further natural and even small reduction of blood pressure can be insufficient and incapable of providing brain protection from ischemic stroke. On the other hand, normalization of cerebral perfusion pressure after reconstructive operation on ICA can lead to development of a hyperperfusion syndrome due to prior persistent vasodilation of microvascular vessels which have lost their ability to adequate vasoconstriction under new hemodynamic conditions.

Today there are two main approaches to studying cerebral blood flow and CVR. The first one is based on estimation of volumetric cerebral blood flow with flowsensitive methods of PET, CT, SPECT, MRI in a perfusion mode and changes of the latter against a background of $\mathrm{CO} 2$ inhalation or IV administration of Diamox (Acetazolamide). However, complexity, inertness and even insecurity of these methods in determining upper and lower thresholds of blood pressure with the purpose of adequate regulation of cerebral blood flow in every individual limit their wide use in routine neurosurgical practice.

The second approach is aimed at studying the first derivative of volume cerebral blood flow, i.e. blood flow velocity (BFV) in major cerebral arteries. It is done with transcranial Doppler sonography (TCD). This method is 
less time-consuming and examination can be carried out just at a patient's bedside. At present the arsenal of methods for studying CVR with the help of TCD is rather diverse and includes estimation of CO2-reactivity in response to Diamox administration, carbogen inhalation, a cuff test, evaluation of slow oscillations of systemic and cerebral hemodynamics with correlation and cross-spectral analyses.

\section{Clinical Course of the Disease and Its Types}

One of the first articles describing use of TCD for CVR estimation was written by E. Ringelstein, et al. [10]. The retrospective analysis of data of 40 cases with ICA occlusion allowed the authors to reveal reliable reduction of CO2-reactivity on the occlusion side $(45.2 \%)$ in contrast to healthy volunteers $(85.6 \%)$. Besides, comparison of the results depending on a type of a stenosis course demonstrated that CO2-reactivity in cases with a symptomatic lesion was reliably lower $(37.6 \%)$ than in asymptomatic patients (62.9\%).

M. Reinhard, et al. [11] did not find out reliable difference between data of the correlation analysis in symptomatic and asymptomatic courses of stenosis. At the same time indices of CO2-reactivity and crossspectral analysis were reliably lower in cases with a symptomatic type of a clinical course.

\section{Stenosis Degree}

R. White, H. Markus [12] used a cuff test for assessing autoregulation in 27 cases with stenosic and occlusive of ICA and 21 healthy individuals. The comparative analysis showed reliable diminution of an autoregulation index in a group of patients $(3.3 \pm 2.2)$ as compared to healthy volunteers $(6.3 \pm 1.1)$. In the authors' opinion, impaired autoregulation can be used for selecting cases with a higher risk of hemodynamic stroke during prescription of hypotensive drugs, planning of an operation and general anesthesia.

Likewise, the transfer function analysis allowed $\mathrm{H}$. $\mathrm{Hu}$, et al. [13] to reveal negative correlation between a rate of autoregulation and a stenosis degree in every frequency range of slow oscillations. A phase shift within the range of Mayer's waves turned out to be the most informative index which is quite compatible with data of other researchers [11].

It should be noted, that M. Reinhard, et al. [14] pointed at possible dependence of CVR impairment on a degree of ICA stenosis and examined cases with stenosis of more than $70 \%$. This supposition was confirmed by S. Tang, et al. [15] studying autoregulation dynamics on the basis of the correlation analysis of data obtained in 21 cases with a degree of ICA stenosis of $\geq 50 \%$. Mean values of correlation index $M_{x}$ in 17 cases with critical stenoses (more than $70 \%$ ) and 8 patients with moderate stenoses (59-69\%) before stenting and after operation were as follows: $0.36 \pm 0.14$ versus $0.22 \pm 0.09$ and 0.36 \pm 0.15 versus $0.20 \pm 0.01$, respectively. Thus, one can see a reliable reduction of $M_{x}$ after surgical intervention and improving of autoregulation in the ipsilateral middle cerebral artery (MCA).

On the contrary, studies by V.B. Semenyutin, et al. [16] refute conclusions of some authors concerning relationship between a stenosis degree and a state of autoregulation. They estimated an autoregulation rate using the cross-spectral analysis of slow oscillations of systemic blood pressure and BFV in the ipsilateral MCA and failed to discover considerable dependence of autoregulation on a degree of ICA stenosis even in cases with critical lesions.

\section{Prognostication of Ischemic Strokes}

The prospective study by B. Kleiser and B. Widder [17] embracing 85 cases with ICA occlusion showed that CO2-reactivity was an informative index for prognostication of stroke development during $38 \pm 15$ months. The authors classified reactivity according to three categories: sufficient, demininshed and exhausted. The rate of acute stroke in the ipsilateral region watched in 37 cases with diminished or exhausted reactivity during the first catamnestic year was more than $20 \%$, whereas 48 patients with sufficient reactivity had no episodes of acute stroke.

The retrospective study by $\mathrm{H}$. Yonas, et al. [18] demonstrated analogous results in 65 cases with symptomatic critical stenosis or ICA occlusion who underwent CT-examination with administration of radio-xenon and a test with Diamox for estimation of volumetric cerebral blood flow. Basic indices of regional cerebral blood flow being lower than $45 \mathrm{ml} / 100 \mathrm{~g} / \mathrm{min}$ and its reduction after Diamox administration for more than $5 \%$ were unfavorable prognostic criteria of stroke development. It was accompanied by an increase of a stroke rate up to $36 \%$.

The prospective study by A. Gur, et al. [19] was carried out on 44 cases with asymptomatic critical ICA stenoses (> 70\%) with the purpose of identifying a prognostic value of CVR and its role in estimating risks of stroke development during 24 months. A growth of $B F V$ in the ipsilateral MCA was calculated by data of TCD after IV administration of $1 \mathrm{~g}$ of Diamox. As for 23 cases without CVR impairment they had no strokes during the follow-up period (BFV in MCA increased for more than $40 \%$ after Diamox administration). At the same time there were 2 strokes and 5 transitory ischemic attacks in 21 cases with asymptomatic critical stenosis and impaired CVR.

\section{Perioperative Dynamics of Cerebrovascular Reserve}

A. Naylor, et al. [20] studied CO2-reactivity by perioperative measurement of volume cerebral blood flow in 69 cases with ICA stenosis using PET. They revealed re- 
liable difference of CVR in cases with symptomatic and asymptomatic courses and its considerable improvement after carotid endarterectomy in symptomatic ICA stenoses. However, the authors came to the conclusion that CVR estimation had no essential effect on longterm results of surgical treatment.

M. Reinhard, et al. [14] assessed autoregulation dynamics in 58 cases with ICA stenosis of more than $70 \%$ after reconstructive operations (carotid endarterectomy or stenting). Indices of CO2-reactivity and dynamic autoregulation increased reliably in both groups of operated cases on the third day after operation.

V.B. Semenyutin, et al. [16] demonstrated a positive effect of reconstructive operations on autoregulation and functional outcomes. However, it was watched only when initial preoperative indices of autoregulation on a lesion side were indicative of its impairment. In case of a normal initial state of autoregulation an operation did not lead to its considerable change and did not improve functional outcomes in cases with symptomatic as well as asymptomatic ICA stenoses. Reconstructive operations in both clinical groups with impaired autoregulation were accompanied by its early restoration (a phase shift increase up to normal values). It did not contradict to literature data $[14,21]$. At the same time some cases with critical ICA stenosis had no autoregulation disorders before planning an operation. Many authors stick to the opinion that despite marked stenosis this "favorable state" of autoregulation appears to be conditioned by sufficient development of collateral circulation [8]. Thus, one can come to the following conclusion: in case of missing autoregulation impairment critical ICA stenosis should not be regarded as a risk factor inevitably leading to cerebral infarction. Optimized drug therapy with dynamic control of a functional state of cerebral hemodynamics in this category of cases would be more preferable than aggressive surgical tactics. First of all it concerns patients with asymptomatic critical ICA stenosis and contradictory indications for surgical treatment which demand further assessment $[2,21]$. According to the latest data, cases with asymptomatic ICA stenosis receiving optimized drug treatment against a background of a natural course of the disease had a much lower risk $(\cdot 1 \%)$ of ipsilateral ischemic stroke development during a two-year period than a risk of postoperative complications arising within 30 days (carotid endarterectomy - $1.4 \%$ and stenting - 2.5\%) [22,23].

\section{Collateral Circulation}

F. Vernieri, et al. [24] carried out the prospective study using Cox regression analysis. It showed that factors reliably increasing a stroke risk included a growing number of pathways of collateral circulation and a state of CO2-reactivity by data of a breathholding test. However, the authors did not discover direct dependence between CVR and an anatomic configuration of collateral circulation. A risk of ischemia development depends not on a type of collateral circulation but on a number of incorporated ways of anastomosing for compensation of cerebral perfusion in a compromised region.

M. Reinhard, et al. [11] consider that CVR disorders in symptomatic stenoses are a more frequent phenomenon when paths of collateral anastomosing are activated. The authors distinguish three types of collateral circulation: type I - via paths of the first order, i.e. anterior and posterior communicating arteries; type II - via secondary paths, i.e. ophthalmic and leptomeningeal arteries of the external carotid artery region or their combination with paths of type I; type III - via stenosed primary paths of the anterior part of the Willis circle. The cross-spectral analysis used for estimation of CO2-reactivity and dynamic autoregulation demonstrated that the most insignificant CVR disorders and the lowest rate of strokes were watched in cases with collateral circulation of type I. On the contrary, gross CVR disorders and a high rate of strokes were typical of patients with type III. Types II and III were more common for symptomatic ICA stenosis.

Reasoning and conclusions of $\mathrm{M}$. Reinhard, et al. [11] are characterized by some discrepancy. On the one hand, a good state of CVR in the ipsilateral region is identified in case of adequate functioning of anterior and posterior communicating arteries (type I). On the other hand, stenosing of anterior paths of the first order leads to gross disorders of vasomotor reactivity and autoregulation. It appears, that distinguishing collateral circulation of type I and II the authors tried to characterize inefficacy of the whole system of cerebral hemodynamics incapable of compensating reduced cerebral perfusion on the side of ICA stenosis via natural paths due to different reasons.

The study by V.B. Semenyutin, et al. [16] did not reveal reliable difference in indices of autoregulation depending on age, gender, a type of an atherosclerotic plaque (ASP), cormobid factors. It makes it possible to conclude that a type of a clinical course and a functional state are determined exactly by compensatory abilities of the system of collateral circulation. Its effective functioning ensures adequate cerebral perfusion in a compromised vascular region despite considerable decrease of blood pressure watched distally from a level of stenosis of a precerebral ICA segment. It allows to minimize a risk of hemodynamic ischemia development. In such cases one can talk about an asymptomatic cerebral hemodynamic model which is not rare in ICA thromboses of various etiology. Furthermore, sometimes a high degree of collaterization of cerebral circulation gives a chance to perform deconstructive operations in case of giant aneurysms, carotid-cavernous fistula, basal tumors growing into ICA and to avoid necessity of applying extra-intracranial anastomoses [24-26]. 


\section{Reperfusion Complications}

One more pressing challenge is studying a role of autoregulation and its state in prognostication of reperfusion complications after reconstructive operation. The rate of a cerebral hyperperfusion syndrome after endarterectomy/stenting is less than $1.9 \%$ [27]. The majority of authors explain its emergence by impaired autoregulation in a postoperative period. Its bedside perioperative assessment provides timely prevention of possible reperfusion complications which are sure to worsen functional outcomes of surgical treatment.

Existing data allow to come to a conclusion that noninvasive estimation of autoregulation with crossspectral analysis of slow oscillations of systemic and cerebral hemodynamics is an important and informative determinant of a stroke risk in cases with ICA stenosis. Revealing patients with impaired autoregulation helps to optimize treatment strategy in symptomatic and asymptomatic stenoses. A risk of stroke development in asymptomatic critical ICA stenosis and a normal autoregulatory response is minimum. It is expedient to use optimized drug therapy in such cases. On the contrary, cases with impaired autoregulation have the greatest risk of stroke development irrespective of a clinical course. Thus, they should be regarded as candidates for surgical treatment.

\section{Atherosclerotic Plaque and Its Embologeneity}

ASP instability is still an unsolved problem conditioning a reliable increase of a stroke risk due to microembolia. Their identification is connected with some difficulties. In particular, microemboli detection on the basis of TCD-monitoring does not always permit to determine their source and a stroke subtype (atherothrombotic, cardioembolic, etc.). It somehow hampers stroke prognostication by criteria of plaque instability and a choice of treatment tactics [3]. Furthermore, some studies demonstrated that use of optimized drug therapy reliably reduced a number of microemboli verified with TCD from $12.6 \%$ up to $3.7 \%[3,28]$. According to J. Spence, et al. [29] optimized drug therapy is a method of the first priority for cases with an asymptomatic course of critical ICA stenosis and microembolia which is not connected with ASP.

As for the study by V.B. Semenyutin, et al. [16] homoor hyperechoic ASP of types I-II on the Gray-Weale scale were diagnosed in the majority of cases. Probably it was that very cause which did not allow the authors to reveal considerable difference in an autoregulation state and its dependence on an ASP type in symptomatic and asymptomatic cases by data of cross-spectral analysis. It can be assumed that autoregulation impairment in instable and heterogeneous ASP (types III-IV) will be more serious as along with hemodynamic factors (decreased perfusion pressure watched distally from stenosis) thromboembolism begins to play a more important role in stroke development. However, this assumption does not conflict with the conclusion that cases with symptomatic stenosis and impaired autoregulation need surgical treatment.

\section{Cognitive Disturbances}

If manifestations of critical stenosis are represented only by cognitive disturbances then a choice of treatment tactics is determined on the basis of additional prognostic criteria [28]. Some authors pay great attention to ultrasonic characteristics of ASP and first of all its embologeneity. Many authors emphasize importance of a degree to which cognitive disorders are marked as well as presence of cormobid factors and ischemic events not only in the region of ICA stenosis but also in other intracranial vascular regions $[3,28,30,31]$. Our own experience makes it possible to state that an initial state of autoregulation in cognitive disturbances is no less important and sometimes can play a key role in choosing treatment modality. From this point of view cases with impaired autoregulation have the greatest probability of hemodynamic stroke development and thus should be regarded as the first-priority candidates for surgical treatment.

This statement is equally true for cases with symptomatic ICA stenosis as according to literature data the majority of patients with verified ipsilateral strokes have CVR impairment. There is no doubt that reconstructive operation in this category of cases are to be performed in the nearest period after a sustained episode [32]. At the same time despite a critical degree of stenosis some cases with verified stroke may have normal indices of autoregulation and minimum manifestations of neurologic symptoms.

\section{Results of Cerebrovascular Reserve Assess- ment and Their Meta-Analysis}

The goal of systematic review and meta-analysis of literature data carried out by A. Gupta, et al. [6] was to assess an informative value of CVR indices from the point of view of prognostication of stroke development in cases with atherosclerotic ICA lesions. They established a reliable relationship between CVR indices on the one hand and a risk of development of stroke/a transitory ischemic attack in cases with occlusive and stenotic ICA lesions on the other both in symptomatic and asymptomatic types of a clinical course.

The authors estimated a prognostic role of $\mathrm{CO}_{2}$ reactivity on the basis of all presented meta-analyses. Today there are no analogous studies highlighting an informative nature of indices of an autoregulation state. However, it is indisputable that TCD used for assessment of a functional state of cerebral hemodynamycs is the most effective method [29] and according to the latest data it makes it possible to optimize economic costs of treatment of cases with ICA stenoses. 


\section{Conclusion}

The undertaken literature review permits to conclude that CVR state in ICA stenoses reliably effects a type of the disease course and a rate of ischemic events. One can see no impairment of this state even in a high degree of stenosis and occlusion in case of sufficient compensation of collateral circulation whose quantitative equivalent it really is. Surgical intervention in initially impaired CVR can lead to its improvement in a postoperative period. Due to this fact, it is possible to assume that disorders of autoregulation and $\mathrm{CO}_{2}$-reactivity may be used as criteria of selecting cases for surgical treatment of both asymptomatic and symptomatic ICA stenoses but only under the condition of low ASP embologeneity.

Taking into account the quality and quantity of accumulated knowledge, reliable conclusions of high practical value and applied methodology studies of CVR in ICA stenoses are not inferior to those carried out in craniocerebral trauma, aneurismal subarachnoid hemorrhages, hemorrhagic strokes and other pathology. Necessity of CVR assessment for improving efficacy of treatment in severe brain injury, cerebral aneurysms, hypertensive hemorrhages is a generally acknowledged fact which is reflected in international guideline recommendations of the leading countries of the world. It should be noted that evidence of results of CVR estimation in the aforesaid pathology is not very high which is explained by an insufficient number of randomized multicenter studies. It equally concerns cases with ICA stenoses. None of the methodological guidelines worked out in the USA, China, South Korea, Germany, Canada, Italy, New Zealand, Russia and other developed countries and devoted to treatment of cases with stenotic and occlusive ICA lesions contain any information on a role of impaired CO2-reactivity and autoregulation in pathogenesis of stroke development as well as on an informative character of indices of CVR state as criteria of determining treatment tactics and outcome prognostication [1,33-36].

Meanwhile the present analytical literature review clearly shows that today there is a great need for carrying out multicenter randomized studies. Their main task is to solve a fundamental problem concerning possible indications for use of CVR assessment in cases with ICA stenoses in order to get pathogenetically substantiated objectivization of efficacy of this or that treatment and its effect on functional outcomes and life quality of cases in the nearest and long-term periods.

\section{Disclosures/Conflict-of-Interest}

The authors declare no conflict of interest.

Source(s) of Support in the Form of Grants, Equipment, Drugs, or all of these

No funding. The authors declare that the research was conducted in the absence of any commercial or financial relationships that could be construed as a potential conflict of interest. No declarations.

\section{References}

1. Abbott AL, Nicolaides A (2015) Improving outcomes in patients with carotid stenosis: Call for better research opportunities and standards. Stroke 46: 7-8.

2. Halliday A, Mansfield A, Marro J, Peto C, Peto R, et al. (2004) Prevention of disabling and fatal strokes by successful carotid end-my in patients without recent neurological symptoms: randomized controlled trial (ACST). Lancet 363: 1491-1502.

3. Gupta A, Kesavabhotla K, Barbadaran H, Kamel H, Pandya A, et al. (2015) Plaque echolucency and stroke risk in asymptomatic carotid stenosis: a systemic review and meta-analysis. Stroke 46: 91-97.

4. Rothweell PM, Eliasziw M, Gutnikov SA, Fox AJ, Taylor DW, et al. (2003) Carotid endarterectomy trialists' callaboration. Analysis of pooled data from randomized controlled trials of endarterectomy for symptomatic carotid stenosis. Lancet 361: 107-116.

5. Fanning JP, Wesley AJ, Wong AA, Fraser JF (2014) Emerging spectra of silent brain infarction. Stroke 45: 34613471.

6. Gupta A, Chazen JL, Hartman M, Delgado D, Anumula N, et al. (2012) Cerebrovascular reserve and stroke risk in patients with carotid stenosis or occlusion: a systematic review and meta-analysis. Stroke 43: 2884-2891.

7. King A, Serena J, Bornstein N, Markus HS, ACES Investigators (2011) Does impaired cerebrovascular reactivity predict stroke risk in asymptomatic carotid stenosis? A prospective substudy of the asymptomatic carotid emboli study. Stroke 42: 1550-1555.

8. Reinhard M, Muller T, Guschlbauer B, Timmer J, Hetzel A (2003a) Dynamic cerebral autoregulation and collateral flow patterns in patients with severe carotid stenosis or occlusion. Ultrasound Med Biol 29: 1105-1113.

9. Schytz HW, Hansson A, Phillip D, Selb J, Boas DA, et al. (2010) Spontaneous low-frequency oscillations in cerebral vessels: applications in carotid artery disease and ischemic stroke. J Stroke Cerebrovasc Dis 19: 465-474.

10. Ringelstein EB, Sievers C, Ecker S, Schneider PA, Otis SM (1988) Noninvasive assessment of CO2-induced cerebral vasomotor response in normal individuals and patients with internal carotid artery occlusions. Stroke 19: 963-969.

11. Reinhard M, Roth M, Muller T, Czosnyka M, Timmer J, et al. (2003b) Cerebral autoregulation in carotid artery occlusive disease assessed from spontaneous blood pressure fluctuations by the correlation coefficient index. Stroke 34: 2138-2144.

12. White RP, Markus HS (1997) Impaired dynamic cerebral autoregulation in carotid artery stenosis. Stroke 28: 13401344.

13. Hu HH, Kuo TB, Wong WJ, Luk YO, Chern CM, et al. (1999) Transfer function analysis of cerebral hemodynamics in patients with carotid stenosis. J Cereb Blood Flow Metab 19: $460-465$.

14. Reinhard M, Roth M, Müller T, Guschlbauer B, Timmer J, et al. (2004) Effect of carotid endarterectomy or stenting on impairment of dynamic cerebral autoregulation. Stroke 35: $1381-1387$ 
15. Tang SC, Huang YW, Shieh JS, Huang SJ, Yip PK, et al. (2008) Dynamic cerebral autoregulation in carotid stenosis before and after carotid stenting. J Vasc Surg 48: 88-92.

16. Semenyutin VB, Asaturyan GA, Nikiforova AA, Aliev VA, Panuntsev GK, et al. (2017) Predictive value of dynamic cerebral autoregulation assessment in surgical management of patients with high-grade carotid artery stenosis. Front Physiol.

17. Kleiser B, Widder B (1992) Course of carotid artery occlusions with impaired cerebrovascular reactivity. Stroke 23: $171-174$

18. Yonas H, Smith HA, Durham SR, Pentheny SL, Johnson DW (1993) Increased stroke risk predicted by compromised cerebral blood flow reactivity. J Neurosurg 79: 483-489.

19. Gur AY, Bova I, Bornstein NM (1996) Is impaired cerebral vasomotor reactivity a predictive factor of stroke in asymptomatic patients? Stroke 27: 2188-2190.

20. Naylor AR, Merrick MV, Sandercock PA, Gillespie I, Allen $P$, et al. (1993) Serial imaging of the carotid bifurcation and cerebrovascular reserve after carotid endarterectomy. $\mathrm{Br} J$ Surg 80: 1278-1282.

21. Paraskevas KI, Nicolaides AN, Veith FJ (2015) Carotid endarterectomy may be required in addition to best medical treatment for some patients subgroups with asymptomatic carotid stenosis. Vascular 23: 62-64.

22. Hart RG, Ng KH (2015) Stroke prevention in asymptomatic carotid artery disease: Revascularization of carotid stenosis is not the solution. Pol Arch Med Wewn 125: 363-369.

23. Spence JD, Pelz D, Veith FJ (2014) Asymptomatic carotid stenosis: Identifying patients of high enough risk to warrant endarterectomy or stenting. Stroke 45: 655-657.

24. Vernieri F, Pasqualetti $P$, Matteis M, Passarelli F, Troisi E, et al. (2001) Effect of collateral blood flow and cerebral vasomotor reactivity on the outcome of carotid artery occlusion. Stroke 32: 1552-1558.

25. Lubicz B, Collignon L, Raphaeli G, Pruvo JP, Bruneau $M$, et al. (2010) Flow-diverter stent for the endovascular treatment of intracranial aneurysms: a prospective study in 29 patients with 34 aneurysms. Stroke 41: 2247-2253.

26. Verhaeghe JL, Montagne S, Belotzerkovski I, Bracard S, Henneton C, et al. (2003) Is carotid resection a valuable option in advanced head and neck squamous cell carcinomas. Bull Cancer 90: 607-613.

27. Moulakakis KG, Mylonas SN, Sfyroeras GS, Andrikopoulos $\checkmark$ (2009) Hyperperfusion syndrome after carotid revascularization. J Vasc Surg 49: 1060-1068.

28. Nicolaides AN, Kakkos SK, Kyriacou E, Griffin M, Sabetai $M$, et al. (2010) Asymptomatic Carotid Stenosis and Risk of Stroke (ACSRS) Study Group. Asymptomatic internal carotid artery stenosis and cerebrovascular risk stratification. J Vasc Surg 52: 1486-1496.

29. Pandya A, Gupta A, Kamel H, Navi BB, Sanelli PC, et al. (2015) Carotid artery stenosis: cost-effectiveness of assessment of cerebrovascular reserve to guide treatment of asymptomatic patients. Radiology 274: 455-463.

30. Abbott AL, Chambers BR, Stork JL, Levi CR, Bladin CF, et al. (2005) Embolic signals and prediction of ipsilateral stroke or transient ischemic attack in asymptomatic carotid stenosis: a multicenter prospective cohort study. Stroke 36: 1128-1133.

31. Demarin V, Zavoreo I, Kes VB (2012) Carotid artery disease and cognitive impairment. J Neurol Sci 322: 107-111.

32. Vandamme H, Limet R (2005) Analysis of pooled data from the randomized controlled trials of endarterectomy for symptomatic carotid stenosis. Rev Med Liege 60: 737-740.

33. Eckstein $H H$, Kühnl $A$, Dörfler $A$, Kopp IB, Lawall $H$, et al. (2013) The diagnosis, treatment and follow-up of extracranial carotid stenosis - a multidisciplinary GermanAustrian guideline based on evidence and consensus. Dtsch Arztebl Int 110: 468-476.

34. Gommans J, Barber P, Fink J (2008) New Zealand guideline for the assessment and management of people with recent transient ischaemic attack (TIA). Stroke Foundation of New Zealand Incorporated, Wellington, New Zealand.

35. Hong KS, Kwon SU, Lee SH, Ko SB, Choi HY (2013) Clinical Research Centre for Stroke. Clinical practice guidelines for stroke. Clinical Research Center for Stroke, Jongu-gu, Seoul, Korea, 76.

36. Setacci C, Argenteri A, Cremonesi A, de Donato G, Galzerano G, et al. (2014) Guidelines on the diagnosis and treatment of extracranial carotid artery stenosis from the Italian Society for Vascular and Endovascular Surgery. J Cardiovasc Surg (Torino) 55: 119-131. 